\title{
Topological Matter and Flat Bands (TMFB)
}

\author{
Pablo D. Esquinazi ${ }^{1}$
}

Published online: 29 January 2018

(C) Springer Science+Business Media, LLC, part of Springer Nature 2018

The papers appearing in this special issue of the Journal of Low Temperature Physics are some of the contributions to the Topological Matter and Flat Bands (TMFB) Conference held in August 17-20, 2017, at the Felix-Bloch-Institute of the Faculty of Physics and Earth Sciences-University of Leipzig, Leipzig, Germany (https:// home.uni-leipzig.de/flatband/). This Conference was a satellite conference of the 28th International Conference on Low Temperature Physics (LT28, http://www.lt28.se) that took place in Gothenburg (Sweden) between August 9-16, 2017.

The TMFB Conference continued the discussion on the importance of flat bands in solid-state physics, which started in Dresden in 2013 (Flat Bands: Design, Topology, and Correlations). It brought together experimentalists and theoreticians to discuss new examples in graphite/graphene and other systems, where the topology and flat bands may play a major role in triggering exceptional phenomena due to extremely singular density of states. The target audience consisted of researchers of graphene/graphite, those studying topological materials, topological superconductivity, interface superconductivity, and also the community of ultracold atom systems. The main topics presented and discussed during the conference included: Lifhshitz transitions, superconductivity/superfluidity in systems with exact or approximate flat bands, two-dimensional superconductivity, nodal topological superconductivity, magnetism and magnetotransport, surface states, topological metals, Weyl fermions and points, transport phenomena, Fano resonances, quantum-Hall systems including superconductors, flat-band phononic lattices, flat bands in strongly interacting systems, as examples. There were 32 oral presentations and 11 posters.

Pablo D. Esquinazi

esquin@physik.uni-leipzig.de

1 Fakultaet fuer Physik und Geowissenschaften, University of Leipzig, Leipzig, Germany 
The organizing committee was integrated by the following scientists: T. Heikkilä (University of Jyväskylä, Finland), A. P. Schnyder (MPI f. Festkörperforschung Stuttgart, Germany), A. Soluyanov (ETH Zurich/Switzerland), P. Törmä (Aalto University, Finland), and G. Volovik (Aalto University, Finland/Landau Institute, Russia).

Following is the list of participants and invited speakers (indicated in bold):

\begin{tabular}{|c|c|c|}
\hline Barzola-Quiquia & José & Universität Leipzig, Germany \\
\hline Bianconi & Antonio & RICMASS, Italy \\
\hline Dolgopolov & Valery $\mathbf{T}$. & Institute of Solid State Physics, Chernogolovka, Russia \\
\hline Drescher & Moritz & Universität Heidelberg, Germany \\
\hline Esquinazi & Pablo & Universität Leipzig, Germany \\
\hline Fulga & Ion Cosma & IFW Dresden, Germany \\
\hline Gühne & Robin & Victoria University of Wellington, New Zealand \\
\hline Heikkilä & Tero & University of Jyväskylä, Finland \\
\hline Hyart & Timo & University of Jyväskylä, Finland \\
\hline Ikegaya & Satoshi & Hokkaido University, Sapporo, Japan \\
\hline Jaworowski & Błażej & Wrocław University of Science and Technology, Poland \\
\hline Kanazawa & Ikuzo & Tokyo Gakugei University, Japan \\
\hline Khodel & Victor & NRC Kurchatov Institute Moscow, Russia \\
\hline Kunst & Flore & Stockholm University, Sweden \\
\hline Kurilovich & Pavel & $\begin{array}{l}\text { Moscow Institute of Physics and Technology (State University), } \\
\text { Dolgoprudny, Russia }\end{array}$ \\
\hline Kurilovich & Vladislav & $\begin{array}{l}\text { Moscow Institute of Physics and Technology (State University), } \\
\text { Dolgoprudny, Russia }\end{array}$ \\
\hline Lötmann & Tomas & Uppsala University,Sweden \\
\hline Menezes & Natalia & Utrecht University, Netherlands \\
\hline Nissinen & Jaakko & Aalto University, Espoo, Finland \\
\hline Nobukane & Hiroyoshi & Hokkaido University, Sapporo, Japan \\
\hline Ojajärvi & Risto & University of Jyväskylä, Finland \\
\hline Park & Hee Chul & Institute for Basic Science, Daejeon, South Korea \\
\hline Peotta & Sebastiano & Aalto University, Finland \\
\hline Pickett & Warren & University of California Davis, USA \\
\hline Precker & Christian & Universität Leipzig, Germany \\
\hline Ramachandran & Ajith & $\begin{array}{l}\text { Center for Theoretical Physics of Complex Systems, Dae- } \\
\text { jeon, Republic of Korea }\end{array}$ \\
\hline Regnault & Nicolas & CNRS, Paris, France \\
\hline Rizzi & Mateo & Johannes Gutenberg University (JGU) Mainz, Germany \\
\hline Rosenow & Bernd & Universität Leipzig, Germany \\
\hline Sato & Masatoshi & Kyoto University, Japan \\
\hline Shaginyan & Vasily & P.N. Lebedev Physical Institute,Moscow, Russia \\
\hline Soluyanov & Alexey & ETH Zurich, Switzerland \\
\hline Tamura & Shun & Nagoya University, Japan \\
\hline Tanaka & Yukio & Nagoya University, Japan \\
\hline Törmä & Päivi & Aalto University, Espoo, Finland \\
\hline Vicencio & Rodrigo & Universidad de Chile, Santiago, Chile \\
\hline Volovik & Grigory & Aalto University, Espoo, Finland \\
\hline Winkler & Georg W. & ETH Zurich, Switzerland \\
\hline Wulayimu & Maimaiti & $\begin{array}{l}\text { Center for Theoretical Physics of Complex Systems, Dae- } \\
\text { jeon, Republic of Korea }\end{array}$ \\
\hline
\end{tabular}

I thank all the members of the organizing committee for the enthusiasm and support in the organization of the conference, the technical support of Ms. Sandy Ehlers and 
Mr. Lukas Botsch, and the partial support of the German Research Society through DFG-Project ES 86/30-1, as well as of the Graduate School "Leipzig School of Natural Sciences-Building with Molecules and Nano-objects" (BuildMona). 